\title{
Gliederungsmöglichkeiten im sandig ausgebildeten Küsten-Holozän Ostfrieslands
}

\author{
Von Karl-Heinz Sindowski, Hannover
}

Mit 4 Abbildungen und 3 Tabellen

Zusammenfassung. Die sandige wie die tonige Wattfazies des Küsten-Holozäns im ostfriesischen Küstengebiet zeigt in ihren Ablagerungen einen zyklischen Aufbau. Die Zyklen in den Ablagerungen der tonigen Wattfazies sind mit biostratigraphischen und geochronologischen Daten belegte lithostratigraphische, die in denen der sandigen Wattfazies nur undatierbare lithostratigraphische Einheiten (Tab. 1).

Zur Erlangung von Altersangaben und damit der Möglichkeit einer zeitlichen Gleichsetzung der Zyklen in sandiger mit denen in toniger Wattfazies werden Kontaktstellen mit biostratigraphisch und geochronologisch datierten Zyklen bzw. Schichten benutzt. Als Ergebnis wird das "Arbeitsmodell“ einer lithostratigraphischen Gliederung der zyklisch aufgebauten Ablagerungen der sandigen Wattfazies vorgelegt, das einen zeitlichen Vergleich mit den Ablagerungen in toniger Wattfazies erlauben soll (Tab. 2).

A bstract. The holocene marine sediments have a cyclic genesis in the East-Frisian coastal region. The different cycles of clay deposits represent lithostratigraphical units (beds), dated by pollen or radiocarbon. The cycles of sand deposits represent lithostratigraphical units without the possibility of dating by pollen or radiocarbon (Tab. 1).

Points of contacting with biostratigraphically or geochronologically dated clay cycles are used for correlation and dating of sand cycles.

The results of investigation permit a lithostratigraphical comparison and a hypothetical order of age for sand cycles (Tab. 2).

\section{Einleitung}

Die Wattfazies des Küsten-Holozäns umfaßt alle rezenten, subfossilen und fossilen Ablagerungen der Nordsee im Gezeitenbereich sowie in den diesem zugehörigen Rinnensystemen (Seegat-Balje-Priel). Sie tritt in toniger („Schlickwatt“) und sandiger („Sandwatt“, „Rinnenfüllungen“) Ausbildung auf. Die Strandsande im Gezeitenbereich werden als Sonderbildung des Sandwattes betrachtet.

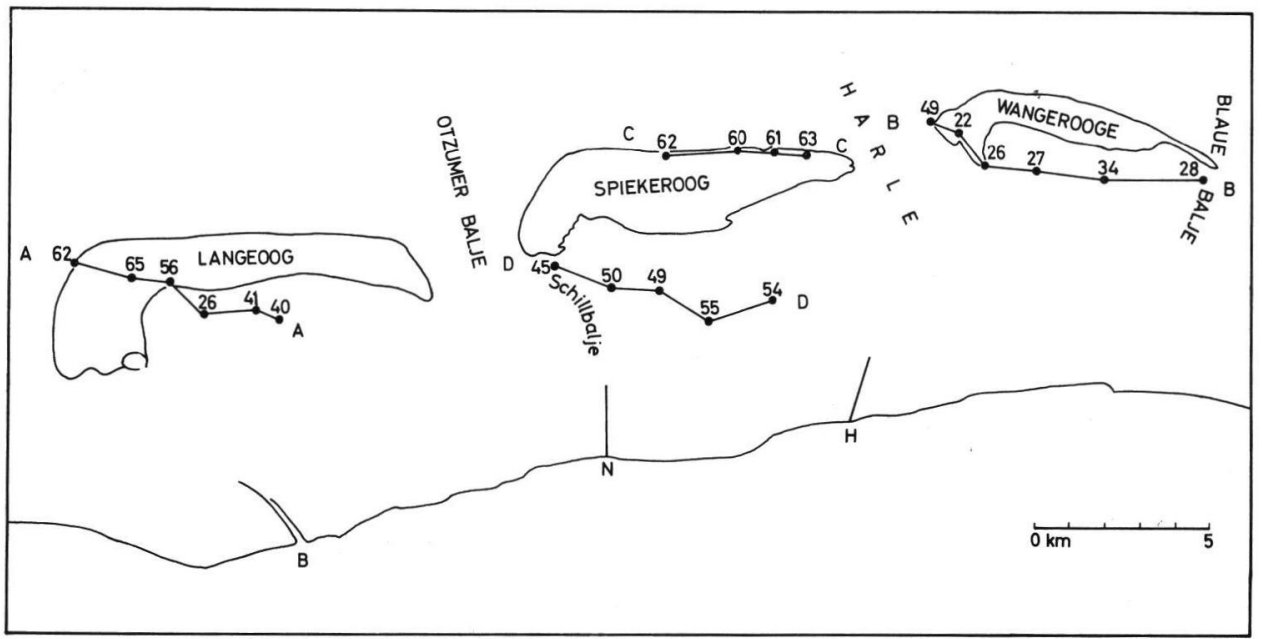

Abb. 1. Lageplan der in Abb. 3 genannten Bohrungen und Schnitte. Die Nummerierung der Bohrungen ist für jede TK 25 gesondert. $\mathrm{B}=$ Bensersiel, $\mathrm{N}=$ Neuharlingersiel, $\mathrm{H}=$ Harlesiel. 
Während die Ablagerungen der tonigen Wattfazies im Gebiet der niederländischen, niedersächsischen und schleswig-holsteinischen Nordseeküste einheitlich gegliedert, lithostratigraphisch eingestuft (Tab. 1) und bereits weitgehend mit biostratigraphischen und geochronologischen Daten belegt sind (BRAND-Hageman-Jelgersma-Sindowski 1966), fehlt bisher für die sandige Wattfazies eine lithostratigraphische Gliederung.

Der Grund hierfür ist das Fehlen datierbarer Horizonte (Torf, Humusdwog) in den Sand-Serien, die eine zeitliche Gleichstellung mit den Klei-Serien erlauben. Da der sandige Wattfazies-Bereich normalerweise dem tonigen räumlich vorgelagert, also küstenferner ist, haben seine Sandfolgen auch keine Verzahnungen mit dem Küstenrandmoor, wie sie die Klei-Serien der tonigen Wattfazies besitzen. Biostratigraphisch (Pollenanalyse) oder geochronologisch (Radiokarbon) datierbare Torfeinlagerungen fehlen folglich. Damit entfällt die wichtigste Grundlage für eine Gliederung der sandigen Wattfazies.

Bekanntlich haben die Ablagerungen der tonigen wie sandigen Wattfazies einen zyklischen Aufbau (Dechend 1957, Sindowski 1957, 1958, 1960, 1964). Ein solcher Sedimentationszyklus stellt eine lithologische Einheit dar und hat einen zweiteiligen Aufbau. Sein unterer Teil zeigt eine Korngrößenzunahme vom Korngrößen-Minimum an der Basis bis

Tabelle 1

Lithostratigraphische Gliederung des niedersächsischen Küstenholozäns in toniger Ausbildung

\begin{tabular}{|c|c|c|c|c|c|}
\hline $\begin{array}{l}\text { Ingressionsphase } \\
\text { n. DECHEND } 1961 \\
\text { W. MüLLER } 1962 \\
\text { SINDOWSKI } 1962\end{array}$ & Schicht & Schichten & $\begin{array}{l}\text { durchschnittl. } \\
\text { Alters-Grenz- } \\
\text { wert nach }{ }^{14} \mathrm{C}\end{array}$ & $\begin{array}{l}\text { Unter- } \\
\text { formation }\end{array}$ & Formation \\
\hline $\begin{array}{l}\mathrm{O}_{4} \\
\mathrm{O}_{3}\end{array}$ & Wyk (W) & \multirow{2}{*}{$\begin{array}{l}\text { Pewsum } \\
(\mathrm{Pe})\end{array}$} & \multirow{2}{*}{ - 1000 n. Chr. } & \multirow[b]{2}{*}{ Dün- } & \multirow{5}{*}{ Holozäne } \\
\hline $\begin{array}{l}\mathrm{O}_{2} \\
\mathrm{O}_{1}\end{array}$ & Tönning ( $\mathrm{T}$ ) & & & & \\
\hline $\mathrm{u}_{2}$ & Schwabstedt (S) & \multirow{3}{*}{$\begin{array}{l}\text { Midlum } \\
\text { (Mi) }\end{array}$} & - 1000 y & \multirow[t]{3}{*}{ kirchen } & \\
\hline & 6. Torf & & \multirow{3}{*}{ - 1700 v. Chr. } & & \\
\hline \multirow[t]{2}{*}{$\mathrm{u}_{1}$} & Meldorf (M) & & & & \\
\hline & 5. Torf & \multirow{4}{*}{$\begin{array}{l}\text { Dornum } \\
\text { (Do) }\end{array}$} & & \multirow{9}{*}{ Calais } & \multirow{3}{*}{ Formation } \\
\hline \multirow[t]{2}{*}{$\mathrm{m}_{3} \mathrm{~B}$} & Husum $(\mathrm{H})$ & & \multirow{3}{*}{ - 2000 v. Chr. } & & \\
\hline & 4. Torf & & & & \\
\hline \multirow[t]{2}{*}{$\mathrm{m}_{3} \mathrm{~A}$} & Fiel $(\mathrm{F})$ & & & & \\
\hline & 3. Torf & \multirow{4}{*}{$\begin{array}{l}\text { Baltrum } \\
\quad(\mathrm{Ba})\end{array}$} & - 2800 v. Chr. & & \\
\hline \multirow[t]{2}{*}{$\mathrm{m}_{2}$} & Eesch (E) & & \multirow{2}{*}{ - 4200 v. Chr. } & & \\
\hline & 2. Torf & & & & \\
\hline \multirow[t]{2}{*}{$\mathrm{m}_{1}$} & Barlt (B) & & \multirow{2}{*}{ - 5500 v. Chr. } & & \\
\hline & 1. Torf & & & & \\
\hline \multicolumn{6}{|c|}{$\begin{array}{l}\text { 1. Torf: spätboreal-frühatlantischer Basalttorf (OvERBECK VII/VIIIa) } \\
\text { 2. Torf: mittelatlantischer Torf (OvERBECK VIIIa/VIIIb) } \\
\text { 3. Torf: spätatlantischer Torf (OvERBECK VIIIb/IX) } \\
\text { 4. Torf: frühsubborealer Torf (OvERBECK IX Mitte) } \\
\text { 5. Torf: mittelsubborealer Torf (OvERBECK IX Mitte) } \\
\text { 6. Spätsubborealer Torf (OvERBECK IX/X) }\end{array}$} \\
\hline
\end{tabular}


zum Korngrößen-Maximum, sein oberer Teil eine Korngrößenabnahme vom KorngrößenMaximum zum Korngrößen-Minimum im Top (Abb. 2 A). Der untere Zyklenteil ist eine Ablagerung der Überflutungsphase oder - in Rinnen - der Durchströmungsphase, der obere Zyklenteil eine der Verlandungsphase oder - in Rinnen - der Verfüllungsphase (Abb. 2 A).

Ursache der Zyklenbildung ist eine kontinuierliche $\mathrm{Zu}$ - und Abnahme der GezeitenStrömungsgeschwindigkeit, die in den Zyklen der tonigen Wattfazies meist gleichzeitig mit einer $\mathrm{Zu}$ - und Abnahme des Salzgehaltes des Wassers verbunden ist.

$\mathrm{Zu}$ - und Abnahme der Gezeiten-Strömungsgeschwindigkeit kann die Folge einer Ingressionsphase der Nordsee sein. Bei der Sedimentation in Rinnen können aber auch andere Ursachen einen meist lokal bedingten Strömungswechsel erzeugen, wie z. B. Eintiefung, Verlagerung und Verfüllung der Seegaten zwischen den Inseln.

\section{Lithostratigraphische Gliederungsmöglichkeiten}

\subsection{Allgemeines}

Die Sedimentationszyklen in den Schichtfolgen der tonigen wie sandigen Wattfazies sind zunächst nur lithologische Einheiten, mit deren Hilfe aber eine Sedimentserie lithostratigraphisch gliederbar ist, wenn folgende Voraussetzungen zutreffen:

1) Der Zyklus muß \pm horizontbeständig und mit Sicherheit in jeder Bohrung wiedererkennbar sein, wenn auch erst durch nachfolgende Laboruntersuchungen (z. B. Korngrößen-Analysen).

2) Der Zyklus muß Merkmale (z. B. Kontakt mit Torfen oder anderen datierbaren Schichten) aufweisen, die Hinweise auf sein relatives oder absolutes Alter geben.

Diese Voraussetzungen erfüllen die Zyklen in den Ablagerungen der tonigen Wattfazies. Durch die einen solchen Zyklus über- und unterlagernden Torfe ist gleichzeitig auch eine biostratigraphische und geochronologische Datierung des zwischenliegenden Zyklus möglich. Die Zyklen in den Ablagerungen der tonigen Wattfazies sind deswegen nicht nur lithologische, sondern auch mit geochronologischen Daten belegte lithostratigraphische Einheiten (Abb. 2 A). Sie bilden jeweils die Ablagerungsfolge einer Ingressionsphase der Nordsee. Da es 6 Ingressionsphasen vor der Zeitwende und 2-4 nach ihr gibt (Tab. 1), müßten auch mindestens 8 Sedimentationszyklen in einem vollständigen Profil der tonigen Wattfazies auftreten.

Da für die Zyklen in den Sedimentserien der sandigen Wattfazies diese Voraussetzungen nicht ohne weiteres zutreffen, müssen andere Kriterien gesucht werden, um entscheiden zu können, ob sie mit den Zyklen in den Ablagerungen der tonigen Wattfazies litho-, bio- oder chronostratigraphisch äquivalent und somit gleichfalls die Ablagerungsfolge je einer Ingressionsphase der Nordsee darstellen. teilt:

Die Sedimentserien der sandigen Wattfazies werden in 3 verschiedene Typen unter-

1) Wattsand-Serien

2) Rinnenfüllungen

2.1) normale kontinuierliche Rinnenfüllungen

2.2) junge Rinnenfüllungen der sich mit den Inseln verlagernden Seegaten und Baljen.

Die Wattsand-Serie besteht normalerweise nur aus 2-3 Sedimentationszyklen, ist fein- bis feinstsandig und hat in ihren Zyklen wenig ausgeprägte Korngrößen-Maxima (Abb. 2 B).

Die normale kontinuierliche Rinnenfüllung besteht aus maximal 8 Zyklen (Zyklus I-VIII). Durch synoptischen Kornkurven-Vergleich (SINDowski 1958) werden grob- bis mittelsandige Rinnenzyklen der Durchströmungsphase mit sehr markant ausgeprägten 

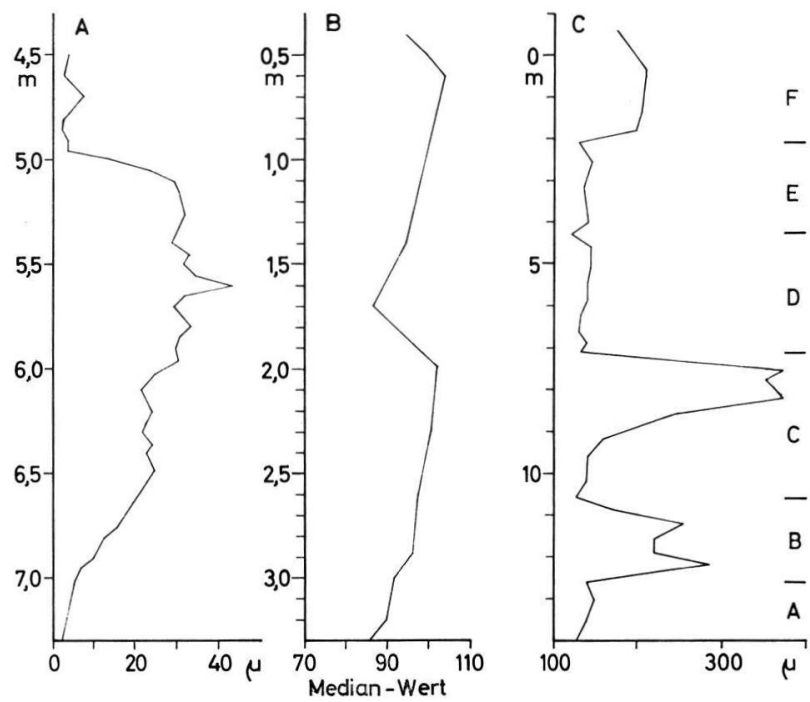

Abb. 2. Sedimentationszyklen. A: Zyklus (Husum-Schicht) in der tonigen Wattfazies. B: Wattsand-Serie mit 2 Zyklen, Bhrg. 21/55 Watt nördl. Bensersiel. C: Rinnenfüllung mit 3 Rinnen- und 3 Verfüllungszyklen, Bhrg. 110/57 Spiekerooger Watt.

Korngrößen-Maxima von mittel- bis feinsandigen Zyklen der Verfüllungsphase mit ausgeprägten Korngrößen-Maxima unterschieden (Abb. 2 C).

Die jungen Verfüllungen der Seegat- und Baljen-Rinnen bestehen aus 4-6 Zyklen (Zyklus 1-6). Durch synoptischen Kornkurven-Vergleich (SINDowski 1958) können ebenfalls Rinnen- und Verfüllungszyklen unterschieden werden. Da die Seegaten in die normalen Rinnenfüllungen eingeschnitten sind und deren obere Zyklen (meist Zyklus IIV) ausgeräumt haben, liegen die jungen Seegat-Zyklen \pm diskordant auf z. T. erheblich älteren Zyklen der normalen Rinnenfüllung (Abb. 3 B-D).

Für lithostratigraphische Gliederungsversuche eignen sich nur die normalen Rinnenfüllungen, weil sie mutmaßlich die gesamte lithostratigraphische Schichtfolge des KüstenHolozäns in toniger Wattfazies umfassen können (Abb. $3 \mathrm{~A}$ ).

Den Sedimentationszyklen wurde bereits früher (SINDOwsKi 1957, 1958, 1960, 1964) ein lithostratigraphischer Wert beigelegt, neuerdings sogar auch den Korngrößen-Maxima der Zyklen (Linke 1965, 1967). Diesem Vorgehen liegen folgende Überlegungen zugrunde:

1) $\mathrm{Da}$ die Zyklen in den Ablagerungen der tonigen Wattfazies nicht nur lithologische, sondern auch mit geochronologischen Daten belegte lithostratigraphische Einheiten sind, deren Tiefenlage vom jeweiligen Nordseespiegelstand bestimmt wurde, könnten analog auch die Zyklen in den Ablagerungen der sandigen Wattfazies - und zwar nur die der normalen Rinnenfüllung - diesen zeitlich entsprechende lithostratigraphische Einheiten und wie diese die Ablagerungsfolge je einer Ingressionsphase der Nordsee sein.

2) Die bei voller Mächtigkeit der normalen Rinnenfüllung vorhandenen 8 Sedimentationszyklen (Abb. $3 \mathrm{~A}$ ) - die gleiche Anzahl, wie in den Ablagerungen der tonigen Wattfazies - treten in nahezu gleicher Tiefenlage in allen untersuchten Profilen des Norderneyer, Baltrumer, Langeooger, Spiekerooger und Wangerooger Wattgebietes auf (Tab. 2, Abb. 4). Daraus ist auf gleiche Sedimentationsverhältnisse in allen, als drenthestadiale Schmelzwassertäler angelegten Rinnen (z. B. Norderneyer, Baltrumer, Langeooger, Harle- und Jade-Rinne) zu schließen. 
3) $\mathrm{D}: \mathrm{e}$ in allen genannten Rinnen fast gleiche Tiefenlage der Sedimentationszyklen (Tab. 2, Abb. 4) deutet zwar nicht, wie die der Zyklen in den Ablagerungen der tonigen Wattfazies, auf eine unmittelbare Abhängigkeit vom jeweiligen Nordseespiegelstand, zeigt jedoch an, daß die bei Rinnen mit stärkerer Wasserfüllung feststellbare indirekte Abhängigkeit vom Meeresspiegelstand besteht. Mit dem im Laufe des Mittelholozäns langsamer ansteigenden Nordseespiegel kann auch die Verfüllung der Rinnen allmählich Schritt halten, so daß die Zyklen IV bis I in ihrer Tiefenlage bereits den äquivalenten Zyklen in den Ablagerungen der tonigen Wattfazies entsprechen.

4) Die Sedimentationszyklen der normalen Rinnenfüllung haben häufig einen ,meist allerdings diskordanten Kontakt mit datierbaren Schichten der tonigen Wattfazies (Abb. $3 \mathrm{~A}-\mathrm{D})$. In den im folgenden diskutierten Beispielen wird anhand von Uber- und Unterlagerung mit lithostratigraphischen Einheiten der tonigen Wattfazies wahrscheinlich gemacht, daß die Zyklen VI und V der normalen Rinnenfüllung den Dornum-Schichten, der Zyklus III den oberen Midlum-Schichten und der Zyklus II den untersten Pewsum-Schichten seitlich äquivalent sind.

\subsection{Lithostratigraphische Gliederungsversuche einzelner Schnitte}

Für lithostratigraphische Gliederungsversuche werden Schnitte durch die Langeooger Rinne (Abb. $3 \mathrm{~A}$ ), die Harle-Rinne (Abb. $3 \mathrm{C}-\mathrm{D}$ ) und die Jade-Rinne (Abb. $3 \mathrm{~B}$ ) ausgewählt. Die Lage dieser Schnitte zeigt Abb. 1.

\section{Schnit t Langeooger Rinne (Langeoog)}

Der Querschnitt durch die verfüllte Langeooger Rinne (Abb. $3 \mathrm{~A}$ ) zeigt, daß die Schichtenfolge der normalen Rinnenfüllung durch kein durchwanderndes Seegat gestört wurde - anhand der paläogeographischen Verhältnisse wird Langeoog als eine alte lagestabile Insel gedeutet (SINDOwSKI 1963) - und 8 Sedimentationszyklen aufweist, von denen der jüngste Zyklus I auf Abb. 3 A nicht dargestellt wurde. Lithostratigraphische Anhaltspunkte im obigen Schnitt sind: 1) diskordante Auflagerung von Zyklus VI auf teilerodierte Baltrum-Schichten der tonigen Wattfazies. Der hangende Torf ist mittelatlantisch und stellt die Basis der Eesch-Schicht dar. 2) Torflage zwischen Zyklus III und II, die aufgrund der Pollendatierung etwa 200 v. Chr. alt ist.

Die lithostratigraphischen altersdatierten Anhaltspunkte machen wahrscheinlich, daß der Zyklus VI dem unteren Teil der Dornum-Schichten (Fiel-Schicht), der Zyklus III dem oberen Teil der Midlum-Schichten (Schwabstedt-Schicht) und der Zyklus II den unteren Pewsum-Schichten (Tönning-Schicht) zeitlich äquivalent ist (Tab. 3).

Schnitt Jade-Rinne (Wangerooge)

Der Querschnitt durch den Westrand der verfüllten Jade-Rinne bei Wangerooge (Abb. 3 B) zeigt einen Aufbau der normalen Rinnenfüllung aus 7 Sedimentationszyklen. Zyklus VIII kam wegen zu hoher Lage der Holozän-Basis nicht zum Absatz. Ostlich Punkt 26 wurde die Rinne von einem jungen Seegat durchwandert, und zwar von der Blauen Balje, die Wangerooge von der einstigen Insel Minsener Oldeooge trennt (Abb. 1).

Der erste lithostratigraphische Anhaltspunkt ist ein Aufschluß unterhalb der Ruine des gesprengten Westturmes am Nordwestrand von Wangerooge. Hier stehen 0,5 m eines insgesamt 1,3 m mächtigen Kleies an, dessen obere Partien eine ehemalige dichte Besiedlung mit Scrobicularia und Cardium (beide in optimaler Größe, doppelklappig und in Lebendstellung) aufweisen. Die ${ }^{14} \mathrm{C}$-Bestimmung von Muscheln der obersten Scrobicularia-Lage ergab ein absolutes Alter von $500 \pm 180$ nach $\mathrm{Chr}$. ( $\mathrm{Hv} 300$ ). Der Klei gehört demnach zum untersten Teil der Pewsum-Schichten (Tönning-Schicht). Folglich ist der den Klei unterlagernde Zyklus III der normalen Rinnenfüllung bereits zu den oberen Midlum-Schichten (Schwabstedt-Schicht) zu stellen. 

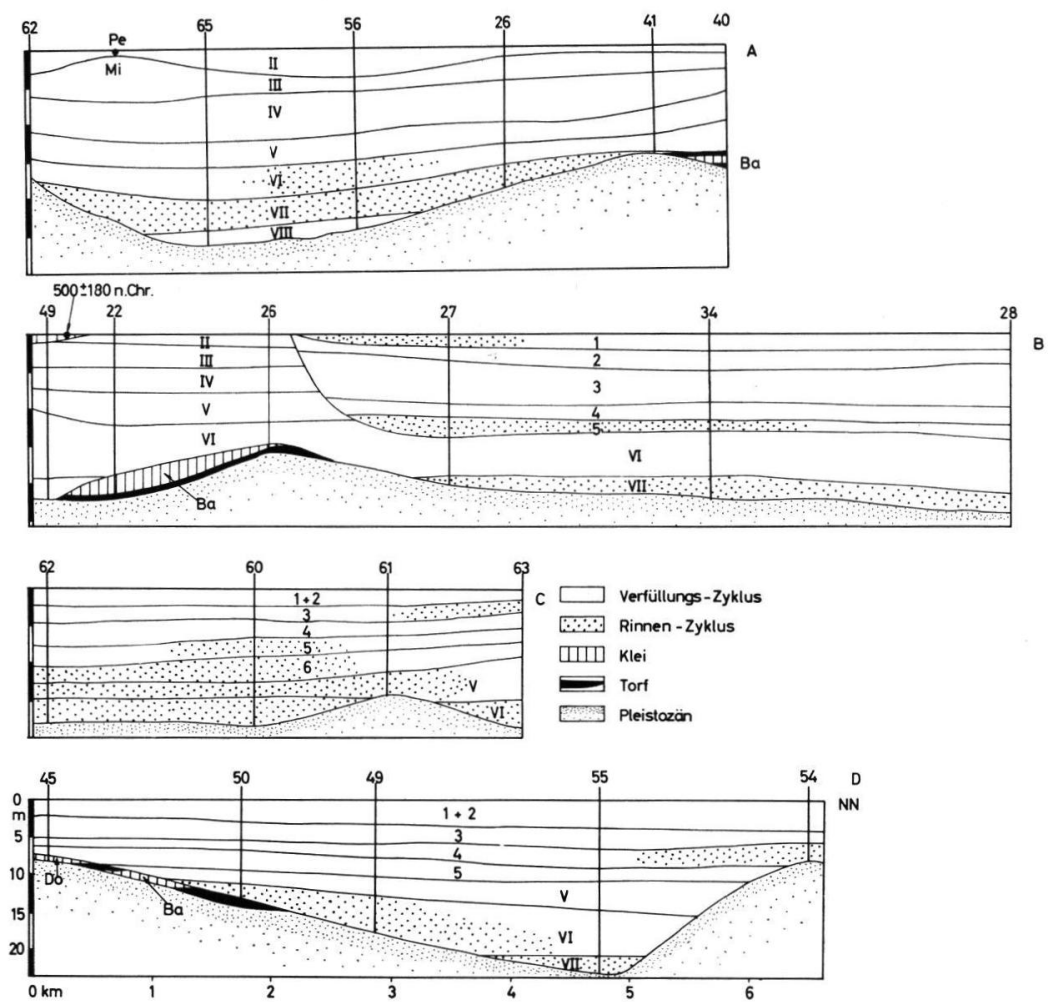

Abb. 3. Schnitte durch Langeoog, Spiekeroog und Wangerooge. I-VIII Zyklen der normalen Rinnenfüllung, 1-6 Zyklen der jungen Seegatfüllung. $\mathrm{Ba}=$ Baltrum-, Do = Dornum-Schichten. A: Langeooger Rinne. B: Westteil der Jade-Rinne. C: Harle-Rinne. D: Harle-Balje. Lage der Schnitte auf Abb. 1.

In den Bohrungen 22 und 26 liegt auf dem Pleistozän ein spätboreal-frühatlantischer Basaltorf und darüber ein an seiner Oberfläche teilerodierter durchwurzelter Klei, der den Baltrum-Schichten entspricht. Angelagert an diesen Klei ist Zyklus VII, diskordant auf ihm liegt der Zyklus VI, der deshalb mutmaßlich in den unteren Teil der DornumSchichten (Fiel-Schicht) zu stellen ist (Tab. 3).

Bei Einhängung der restlichen Sedimentationszyklen zwischen die beiden lithostratigraphischen Fixpunkte ergibt sich das gleiche "lithostratigraphische“ Bild wie aus dem Langeooger Schnitt.

Die Füllung der jungen Seegatrinne (Blaue Balje) besteht aus 5 Zyklen. Der unterste Zyklus 5 der jungen Seegatrinnenfüllung liegt diskordant als Rinnenzyklus der Durchströmungsphase auf dem teilerodierten Zyklus VI der normalen Rinnenfüllung. Darüber folgen 4 Zyklen (Zyklus 1-4) der Verfüllungsphase, von denen der Zyklus 3 besonders mächtig ist. Trotz Vorhandenseins von topographischen Altkarten können die Zyklen altersmäßig nicht festgelegt werden. Die Punkte 27, 34, 28 lagen seit 1650 ständig auf Wattflächen im Schutze der Insel Wangerooge.

$\mathrm{Schnitt} \mathrm{Harle}-\mathrm{R}$ in ne (S piekeroog)

Einen Teilschnitt durch die verfüllte Harle-Kinne żèż Abb. 3 C. Dieser Schnitt geht durch die heute mit Primärdünen bedeckte Ostplate von Spiekeroog. Die Schichtenfolge der normalen Rinnenfüllung ist durch das durchwandernde Harle-Seegat gestört. Seine Wanderbahn ist zwischen Bohrung 62 und 60 durch den Rinnenzyklus 6 markiert. Im 
Gebiet der Bohrungen 61 und 63 verlagerte sich die Durchströmungsphase des Seegats in immer höher gelegene Zyklen, d.h. das Seegat verflachte sich allmählich, wie auch von den topographischen Altkarten ablesbar ist. Um 1650 lagen die Punkte 62, 60, 61 im Harle-Seegat, der Punkt 63 auf einer Sandplate im Seegat. Um 1750 war der Bereich des Schnittes weitgehend verlandet und Punkt 63 lag bereits am Westrand des HarleSeegats.

Diese aus den topographischen Altkarten entnehmbaren Verhältnisse dürften sich für den Zeitraum 1600-1650 in Zyklus 4, für den 1650-1750 in Zyklus 3 widerspiegeln. Demnach scheint die Altersstellung der jungen Zyklen in der Harle-Seegatrinne folgende zu sein:

$$
\begin{array}{cll}
\text { Zyklus } 1+2 & \text { nach } 1750 \\
\Rightarrow 3+4 & : & 1650-1750 \\
\Rightarrow 5 & : & \text { vor } 1600 \\
\Rightarrow 6 & :
\end{array}
$$

Unter den 6 "Zyklen der jungen Seegat-Rinnenfüllung liegen die Zyklen V-VI der normalen Rinnenfüllung. Die Zyklen VII und VIII kamen hier wegen Hochlage der

\begin{tabular}{|c|c|c|c|c|}
\hline \multirow[t]{2}{*}{ Zyklus } & \multirow{2}{*}{$\begin{array}{c}\text { Zyklen- } \\
\text { Oberkante } \\
\text { (m unter NN) }\end{array}$} & \multirow{2}{*}{$\begin{array}{c}\text { Lithostratigraphische } \\
\text { Anhaltspunkte } \\
\text { der tonigen Wattfazies }\end{array}$} & \multicolumn{2}{|c|}{$\begin{array}{l}\text { Vermutete litho- } \\
\text { stratigraphische Einheiten }\end{array}$} \\
\hline & & & Schicht & Schichten \\
\hline I & & & Wyk & \multirow[b]{2}{*}{ Pewsum } \\
\hline II & ca. 0 & $\begin{array}{l}\text { Baltrum: auf Midlum-Schichten } \\
\text { Langeoog: auf Midlum-Schichten } \\
\text { Spiekeroog: - } \\
\text { Wangerooge: Tönning-Schichten }\end{array}$ & Tönning & \\
\hline III & $2-4$ & $\begin{array}{l}\text { Baltrum: an Dornum-Schichten } \\
\text { Langeoog: - } \\
\text { Spiekeroog: auf Dornum-Schichten } \\
\text { Wangerooge: - }\end{array}$ & Schwabstedt & \multirow{2}{*}{ Midlum } \\
\hline IV & $5-7$ & $\begin{array}{l}\text { Baltrum: an Dornum-Schichten } \\
\text { Langeoog: - } \\
\text { Spiekeroog: auf Dornum-Schichten } \\
\text { Wangerooge: - }\end{array}$ & Meldorf & \\
\hline V & $9-11$ & $\begin{array}{l}\text { Norderney: auf Baltrum-Schichten } \\
\text { Baltrum: auf und an Dornum-Sch. } \\
\text { Langeoog: auf Baltrum-Schichten } \\
\text { Spiekeroog: an Dornum-Schichten } \\
\text { Wangerooge: - }\end{array}$ & Husum & \multirow{2}{*}{ Dornum } \\
\hline VI & $13-15$ & $\begin{array}{l}\text { Norderney: an Baltrum-Schichten } \\
\text { Baltrum:- - } \\
\text { Langeoog: auf Baltrum-Schichten } \\
\text { Spiekeroog: auf Baltrum-Schichten } \\
\text { Wangerooge: an Baltrum-Schichten }\end{array}$ & Fiel & \\
\hline VII & $18-20$ & $\begin{array}{l}\text { Baltrum: - } \\
\text { Langeoog: an Baltrum-Schichten } \\
\text { Spiekeroog: - } \\
\text { Wangeroog: an Baltrum-Schichten }\end{array}$ & Eesch & \multirow[t]{2}{*}{ Baltrum } \\
\hline VIII & $23-24$ & & Barlt & \\
\hline
\end{tabular}

Tabelle 2

Vermutetelithostratigraphische Stellung der Sedimentationszyklen der normalen Rinnenfüllung 
Holozän-Basis nicht zum Absatz. Entsprechend den Zyklen der normalen Rinnenfüllung aus der Langeooger und Jade-Rinne sowie aus der Harle-Baljenrinne (Abb. 3 A, B, D) könnte Zyklus VI den unteren Dornum-Schichten entsprechen. Lithostratigraphische Anhaltspunkte fehlen, sind jedoch aus der Umgebung der Schnittlinie bekannt (Abb. $3 \mathrm{D}$ ).

Schnitt Harle-Balje (Spiekerooger Wat t)

Zur Überprüfung der Gliederung der Sandfolgen im Querschnitt durch das HarleSeegat (Abb. 3C), wo lithostratigraphische Anhaltspunkte fehlen, søll ein südlicher gelegener Querschnitt durch die verfüllte Harle-Rinne (Abb. $3 \mathrm{D}$ ) dienen. Die junge Seegat-Füllung des Harle-Seegats muß sich auch im oberen Teil der Verfüllung der HarleBalje bemerkbar machen, da diese dem Harle-Seegat tributär war. Außerdem muß der obere Teil der Verfüllung auch entsprechend der fortschreitenden Verlandung und Bedeichung der am Oberlauf der Harle-Balje gelegenen Harle-Bucht recht jugendlich sein.

Aus den topographischen Altkarten ist bekannt, daß um 1650 die Punkte 45, 50, 49, 54 auf Wattflächen lagen, Punkt 55 dagegen in der Harle-Balje. Um 1750 lagen die Punkte 50, 49 auf Wattflächen, 55 und 54 an Prielrändern und 45 am Rande der Schillbalje (Lage s. Abb. 1). Um 1860 lag Punkt 49 auf der Wattfläche, die Punkte 45 und 50 am Ostrand der Schillbalje und die Punkte 55 und 54 in kleinen Prielen.

Die mit dem Harle-Seegat zusammenhängende junge Rinnenfüllung der Harle-Balje hat im Gegensatz zum Harle-Seegat nur 5 Zyklen. Die Grenze zwischen Zyklus 1 und 2 ist undeutlich.

Unter den 5 Zyklen der jungen Baljenfüllung liegen noch 3 Zyklen der normalen Rinnenfüllung, und zwar die Zyklen VII bis V. Lithostratigraphische Anhaltspunkte sind folgende: 1) in Bohrung 50 liegt der Zyklus VI auf teilerodiertem mittelatlantischem Torf der Baltrum-Schichten. 2) in Bohrung 45 liegt der Zyklus V diskordant auf spätatlantischem Torf der Grenze Baltrum-/Dornum-Schichten. Demnach könnte Zyklus VI den unteren Dornum-Schichten (Fiel-Schicht) und Zyklus V den oberen Dornum-Schichten (Husum-Schicht) zeitlich äquivalent sein (Tab. 3).

\section{Lithostratigraphische Folgerungen}

Die Ergebnisse der geologischen Kartierung der GK 25 Altenwalde, Cuxhaven, Otterndorf und Neuhaus/Oste (SINDOwSKI 1963-1967) haben bereits gezeigt, daß die mächtigen holozänen Sandfüllungen der aus der Hadeler Bucht kommenden tiefen Rinnen lithostratigraphisch gliederbar und mit den Schichtfolgen der tonigen Wattfazies parallelisierbar sind (SINDOwSKI 1968). Da sich hier im Unterelbegebiet die tonige mit der sandigen Wattfazies des Küsten-Holozäns randlich verzahnt sowie Wechsellagen mit dieser bildet, sind zahlreiche datierte lithostratigraphische Anhaltspunkte vorhanden.

Durch die geologische Kartierung der GK 25 Baltrum und Langeoog (BARCKHAusen 1966), Spiekeroog und Wangerooge (Sindowski 1962, 1966) wurde auch die Frage nach einer Gliederungsmöglichkeit der mächtigen sandigen Rinnenfüllungen im Gebiet der ostfriesischen Küste vordringlich.

Nachdem der zyklische Aufbau der tonigen wie der sandigen Wattfazies erkannt war (Abb. 2), lag es nahe, den lithologischen Einheiten der Sedimentationszyklen auch lithostratigraphischen Wert beizumessen. Die Zyklen der tonigen Wattfazies sind datierbare lithostratigraphische Einheiten, die bereits biostratigraphisch und geochronologisch gliederbar sind. Weil jedoch die Zyklen der sandigen Wattfazies keine datierbaren Zwischenlagen haben, entfällt bei ihnen die wichtigste Grundlage für ihre litho- und biostratigraphische sowie vor allem geochronologische Gliederung.

Aus Analogiegründen wurde bereits schon früher vermutet (SINDOwSKI 1957, 1960, 1964), daß auch die Zyklen der sandigen Wattfazies lithostratigraphische Einheiten sind, also jeder Zyklus die Ablagerungsfolge einer Ingressionsphase der Nordsee darstellt. Da 


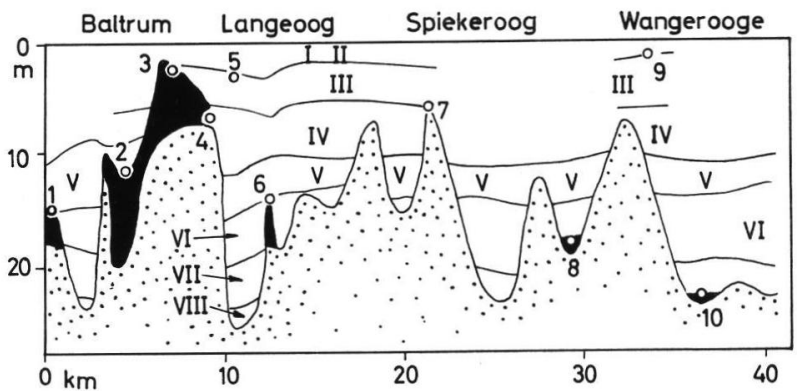

Abb. 4. Vereinfachter Schnitt von Baltrum bis Wangerooge. Tiefenlage der Zyklen I-VIII der normalen Rinnenfüllung. Kontaktpunkte: 1 ob. Baltrum-Sch., 2 unt. Dornum-Sch., 3 Midlum-Sch., 4 ob. Dornum-Sch., 5 Grenze Pewsum/Midlum-Sch., 6 ob. Baltrum-Sch., 7 ob. Dornum-Sch., 8 Baltrum-Sch., 9 Grenze Pewsum/Midlum-Sch., 10 Baltrum-Sch.

in der tonigen wie der sandigen Wattfazies maximal 8 Sedimentationszyklen auftreten, scheint die Analogie vollkommen.

$\mathrm{Da}$ jedoch ein Beweis für die Richtigkeit dieser Vermutung mangels eingeschalteter datierbarer Horizonte nicht erbracht werden kann, mußte ein Umweg über Datierungsmöglichkeiten durch Kontakt mit biostratigraphisch oder geochronologisch fixierten lithostratigraphischen Schichten der tonigen Wattfazies gewählt werden. In vielen Profilen treten solche Kontakte mit der tonigen Wattfazies auf, sei es durch Verzahnung, Unteroder Überlagerung beider Ausbildungen.

Wenn auch durch dieses „Konnektierungsverfahren" keine exakten Grundlagen für eine lithostratigraphische, dem Einteilungsschema der tonigen Wattfazies entsprechende Gliederung erbracht werden können, kann es jedoch das „Arbeitsmodell“ einer lithostratigraphischen Unterteilung liefern.

Tabelle 3

Mutmaßliche lithostratigraphische Stellung der Zyklen in den Schnitten durch die Langeooger, Harle- und Jade-Rinne

\begin{tabular}{|c|c|c|c|}
\hline Zyklus & $\begin{array}{c}\text { Datierter lithostratigraphischer } \\
\text { Anhaltspunkt }\end{array}$ & Schicht & Schichten \\
\hline I & & Wyk & \multirow{2}{*}{ Pewsum } \\
\hline II & $\begin{array}{l}\text { Langeoog: Tönning-Schichten } \\
\text { Wangerooge: Tönning-Schichten }\end{array}$ & Tönning & \\
\hline III & $\begin{array}{l}\text { Langeoog: unter Tönning-Schichten } \\
\text { Wangerooge: unter Tönning-Schichten }\end{array}$ & Schwabstedt & \multirow{2}{*}{ Midlum } \\
\hline IV & & Meldorf & \\
\hline $\mathrm{V}$ & Spiekeroog: auf Fiel-Schichten & Husum & \multirow{2}{*}{ Dornum } \\
\hline VI & $\begin{array}{l}\text { Langeoog: auf Baltrum-Schichten } \\
\text { Spiekeroog: auf Baltrum-Schichten } \\
\text { Wangerooge: auf Baltrum-Schichten }\end{array}$ & Fiel & \\
\hline VII & & Eesch & \multirow{2}{*}{ Baltrum } \\
\hline VIII & & Barlt & \\
\hline
\end{tabular}


Werden alle - nicht nur die hier diskutierten - sedimentologisch untersuchten Profile aus dem ostfriesischen Küstengebiet überschauend betrachtet, so ergibt sich folgendes Bild (Abb. 4):

1) Über biostratigraphisch oder geochronologisch datierten Kleien und Torfen der Midlum-Schichten liegen maximal 2 Zyklen der normalen Rinnenfüllung (Zyklus I + II), die also den Pewsum-Schichten äquivalent zu sein scheinen.

2) Uber entsprechend datierten Kleien und Torfen der Dornum-Schichten liegen, je nach Alter und Tiefenlage (Fiel- oder Husum-Schicht), maximal 5 Zyklen. Demnach könnte Zyklus V den oberen Dornum-Schichten, Zyklus IV und III den Midlum-Schichten entsprechen.

3) Uber entsprechend datierten Kleien und Torfen der Baltrum-Schichten liegen maximal 7 Zyklen der normalen Rinnenfüllung. Zyklus VII könnte demnach den oberen Baltrum-Schichten, Zyklus VI den unteren Dornum-Schichten gleichwertig sein. Zyklus VIII, häufig direkt auf spätboreal-frühatlantischem Basaltorf auflagernd und nur bei besonderer Tiefenlage der Holozän-Basis ausgebildet, würde dann das Äquivalent der unteren Baltrum-Schichten sein.

\section{Schrifttum}

Brand, G., Hageman, B. P., Jelgersma, S. \& Sindowski, K. H.: Die lithostratigraphische Unterteilung des marinen Holozäns an der Nordseeküste. Geol. Jb. 82, 365-384, Hannover 1966.

Dechend, W.: Der Ablauf der holozänen Nordsee-Transgression im oldenburgisch-ostfriesischen Raum, insbesondere im Gebiet von Jever i. O. Geol. Jb. 72, 295-314, Hannover 1957. - - Eine Gliederung des Holozäns an der südlichen Nordseeküste. Z. deutsch. geol. Ges. 112, 524-525, Hannover 1961.

LiNKE, G.: Vorläufige Ergebnisse der Untersuchungen im Neuwerk-Scharhörner Watt. Vortragsref. 32. Tag. Arbeitsgem. nordwestdeutsch. Geol. Hamburg 1965 - - Gliederungsmöglichkeiten im sandigen Küstenholozän. Vortragsref. 34. Tag. Arbeitsgem. nordwestdeutsch. Geol. Husum 1967.

LütTIG, G.: Vorschläge für eine geochronologische Gliederung des Holozäns in Europa. Eiszeitalt. u. Gegenw. 11, 51-63, Öhringen 1960. - - Prinzipielles zur Quartärstratigraphie. Geol. Jb. 82, 177-202, Hannover 1964. - - Der Stand der Holozän-Forschung in Westdeutschland. Rep. VI. Inqua-Congr. 1 (1965), 447-461, Warschau 1966.

Müller, W.: Der Ablauf der holozänen Meerestransgression an der südlichen Nordseeküste und Folgerungen in bezug auf eine geochronologische Holozängliederung. Eiszeitalt. u. Gegenw. 13, 197-226, Öhringen 1962.

SindowsKI, K. H.: Die geologische Entwicklung des Wattengebietes südlich der Inseln Baltrum und Langeoog. Jber. Forschungsst. Norderney 8, 11-35, Norderney 1957. - - Die synoptische Methode des Kornkurven-Vergleiches zur Ausdeutung fossiler Sedimentationsräume. Geol. Jb. 73, 255-275, Hannover 1958. - - Die geologische Entwicklung des Spiekerooger Wattgebietes im Quartär. Jber. Forschungsst. Norderney 11 11-20, Norderney 1960. - - Nordseevorstöße und Sturmfluten an der ostfriesischen Küste seit 7000 Jahren. Geogr. Rundsch. 14, 322-329, Braunschweig 1962. - - Zur Frage der Ostwanderung aller ostfriesischen Inseln. Geogr. Rundsch. 15, 448-455, Braunschweig 1963. - - Die geologische Entwicklung des Wangerooger Wattes im Quartär. Jber. Forschungsst. Norderney 14, 11-20, Norderney 1964. - - Das Watt-Holozän der Oxstedt-Berenscher Rinne am Westrand der Altenwalder Geest südlich Cuxhaven. Z. deutsch. geol. Ges. 115, 167-176, Hannover 1965. - - Das Küsten-Holozän im nördlichen Teil der Hadeler Bucht (Raum Cuxhaven-Otterndorf). Ber. naturhist. Ges. Hannover Beih. 5, Hannover 1968 (im Druck).

Manuskr. eingeg. 1. 5. 1968.

Anschrift des Verf.: Dr. K. H. Sindowskị, Niedersächsisches Landesamt für Bodenforschung: 3 Hannover-Buchholz, Alfred-Bentz-Haus. 